\title{
Prostaglandin Biosynthesis Can Be Triggered by Lipid Peroxides
}

\author{
MARTIN E. HEMLER, HAROLD W. COOK, ${ }^{1}$ AND WILLIAM E. M. LANDS ${ }^{2}$ \\ Department of Biological Chemistry, The University of Michigan, Ann Arbor, Michigan 48109
}

Received August 11, 1978; revised October 26, 1978

\begin{abstract}
Studies of ferriheme cyclooxygenase, using two different assay systems, show that a variety of peroxides can trigger a rapid acceleration of cyclooxygenase activity to produce prostaglandins. Lipid hydroperoxides formed by lipoxygenase were the most potent activators tested, followed by prostaglandin $G_{\mathbf{3}}$, which was slightly less potent. Peroxides nonspecifically generated during arachidonate autoxidation were as potent as the enzymatically formed lipid peroxides. These findings have important implications for cell function since any process which generates peroxides may activate the cyclooxygenase. Thus the balance between formation and removal of cellular lipid peroxides sets a peroxide tone that can regulate the rate of prostaglandin formation in cells.
\end{abstract}

Fatty acid oxygenation catalyzed by cyclooxygenase is the initial, rate-limiting step in the conversion of free fatty acid to prostaglandins, thromboxanes, and prostacyclin. The appearance of these potent physiological agents appears to be strictly regulated since they occur at much lower levels in vivo than predicted from in vitro assays of total biosynthetic capacity $(1,2)$. Inhibition of cyclooxygenase by glutathione peroxidase $(3,4)$ provided the initial evidence that cyclooxygenase catalysis requires some peroxide which apparently could be generated by cyclooxygenase itself (5). Recently the cyclooxygenase product, $\mathrm{PGG}_{2}$ (but not $\mathrm{PGH}_{2}$ ), ${ }^{3}$ has been characterized as the cyclooxygenase-produced activating agent (6). A low level of activating peroxide (estimated at $0.1 \mu \mathrm{M}$ for the crude enzyme) is continuously required during catalysis, and a mechanism was proposed involving a saturable activator site on the enzyme (7). Since preliminary experiments suggested that peroxides other than $\mathrm{PGG}_{2}$ may activate, a comparative study of hydroperoxides and peroxides was made to dis-

\footnotetext{
1 Present address: Department of Pediatrics, Dal housie University, Halifax, Nova Scotia, Canada.

2 To whom requests for reprints may be sent.

${ }^{3} \Lambda$ bbreviations used: PG, prostaglandin; DDC, sodium diethyldithiocarbamate.
}

cover the range of compounds capable of regulating prostaglandin biosynthesis.

\section{MATERIALS AND METHODS}

Purified cyclooxygenase was obtained as previously described $(8,9)$ and excess heme was removed by chromatography on diethylaminoethyl cellulose at pH 7.0 in $20 \%$ glycerol, $1.0 \mathrm{~mm}$ phenol, and $0.02 \mathrm{M}$ potassium phosphate. Using ovine vesicular glands provided by the Upjohn Company, Kalamazoo, Michigan, crude acetone powder preparations of cyclooxygenase were prepared as previously described (10) and activated by treatment with $0.67 \mathrm{mM}$ phenol for $30 \mathrm{~min}$ at $25^{\circ} \mathrm{C} \mathrm{(4)}$. Unsaturated fatty acids were purchased from Nuchek Prep, Elysian, Minnesota, and arachidonic acid was purchased from Anspec Company, Ann Arbor, Michigan. Sodium diethyldithiocarhamate and soybean lipoxygenase were from Sigma Chemical Company, St. Louis, Missouri, and 5,5'-dithiobis(2-nitrobenzoic acid), cumene hydroperoxide, and t-butyl hydroperoxide were from Aldrich, Milwaukee, Wisconsin. The endoperoxide $\mathrm{PGG}_{2}$ was kindly supplied by G. Graff, University of Minnesota.

Oxygenation was monitored polarigraphically as described previously (11) with a constant temperature of $30 \pm 0.1^{\circ} \mathrm{C}$ maintained with a Lauda $\mathrm{K}-2 / \mathrm{R}$ water bath (Brinkman Instruments). Instantaneous velocities were obtained directly with the help of an electronic differentiator (12), and for very fast reactions, the chart recorder was run at top speed $(10 \mathrm{~cm} / \mathrm{min})$.

Lipid hydroperoxides were generated by incubation of aqueous suspensions of the fatty acids with soybean lipoxygenase, and oxygen consumption during the 
reaction was monitored to determine the extent of oxygenation of the substrate. Reaction mixtures were extracted three times with diethyl ether (5), and the lipid extracts dissolved in pentane or acetone. After suspending aliquots of the organic extract in $0.1 \mathrm{M}$ Tris-chloride at $\mathrm{pH} 8.0-8.5$, the concentration of lipid peroxide was determined using the thionitrobenzoate anion reduction assay described previously (13). The $\mathrm{PGG}_{2}$ solutions (stored in ethyl acetate at $-70^{\circ} \mathrm{C}$ ) were dried under nitrogen in small aliquots, made aqueous in $0.1 \mathrm{M}$ Tris-chloride ( $\mathrm{pH} 8.5$ ), and used immediately (within $1 \mathrm{~min}$ ). To measure lipid peroxide formed during autoxidation of $14 \mathrm{mM}$ arachidonate, the colorimetric assay was modified by adding $0.1 \%$ Tween 40 to reduce turbidity, and the thionitrobenzoate anion was measured at $460 \mathrm{~nm}$, using an extinction coefficient of $3060 \mathrm{M}^{-1} \mathrm{~cm}^{-1}$.

Extraction and identification of oxygenated products and the determination of the stoichiometry of oxygen addition to arachidonic acid were carried out as previously described (9) using 3-min incubations of enzyme and substrate.

\section{RESULTS}

In previous studies, demonstration of the requirement for peroxide was facilitated by using inhibitors which either interfered with the activation step, or directly reduced the endogenous level of activating peroxide. Thus during the initial accelerative phase of the reaction, the product peroxide was slow to accumulate and the lag

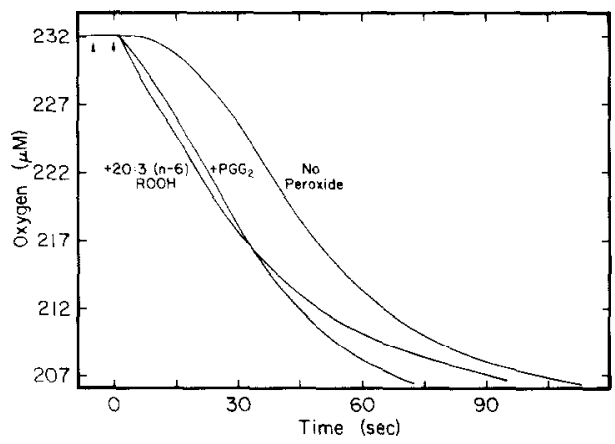

FIG. 1. Accelerated oxygenation rates in the presence of lipid hydroperoxides. Oxygenation chambers contained $3 \mathrm{ml}$ of $0.1 \mathrm{M}$ glycine- $\mathrm{NaOH}(\mathrm{pH} \mathrm{9.5),} 0.67$ $\mathrm{mM}$ phenol, and $50 \mu \mathrm{M}$ 20:4 together with $5 \mathrm{~mm}$ DDC preincubated for 3 min. Cyclooxygenase [ $43 \mathrm{nM}$ bound heme with $61 \mathrm{nM}$ subunit, based on 70,000 daltons per subunit (28)] was added (first arrow), and after about $5 \mathrm{~s}, 10-15 \mu \mathrm{l}$ of $0.1 \mathrm{M}$ Tris-chloride, $\mathrm{pH} 8.5$, was added (second arrow) with or without the indicated peroxide at a final concentration of $1 \mu \mathrm{M}$.

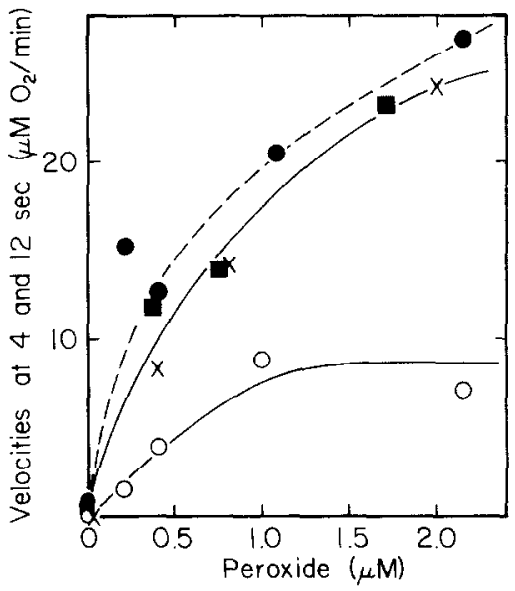

FIG. 2. Comparison of the effect of $\mathrm{PGG}_{2}$ and lipid hydroperoxide on cyclonxygenase oxygenation rates. Using conditions described for Fig. 1, varying concentrations of $\mathrm{PGG}_{2}(\mathrm{O}-\mathrm{O})$, hydroperoxy-20:3(n-6) ( $-\mathbf{E})$, and hydroperoxy-20:4(n-6) $(\times-\times)$ or a buffer blank were added, and the reaction rates were determined at $4 \mathrm{~s}$. For $\mathrm{PGG}_{2}$ reactions, the rates were also determined after $12 \mathrm{~s}$ (

phase allowed time for the addition of peroxide which could trigger a more rapid accleration. For this reason, oxygenations by purified ferriheme cyclooxygenase were carried out at $\mathrm{pH} 9.5$ in the presence of $0.67 \mathrm{~mm}$ phenol, $50 \mu \mathrm{M} 20: 4$, and $5 \mathrm{~mm}$ DDC. Lags were not appreciable unless peroxides were removed from the $20: 4$ by preincubation with DDC for an optimum of $3 \mathrm{~min}$. Using these assay conditions, maximal oxygenation rates were preceded by a significant lag phase (Fig. 1) which was eliminated by the addition of hydroperoxy-20:3 $(n-6)$ or $\mathrm{PGG}_{2}$. The results illustrate that the hydroperoxy-20:3 was at least equal to, and apparently even more effective than, the $\mathrm{PGG}_{2}$. To further compare the stimulatory effect of $\mathrm{PGG}_{2}$ (an endoperoxide-hydroperoxide) with other lipid hydroperoxides, several concentrations of the hydroperoxy derivatives of $20: 3$ and $20: 4$ were examined in reactions similar to those shown in Fig. 1. For simplicity, the reaction velocity at $4 \mathrm{~s}$ after the addition of peroxide was selected as a convenient parameter to describe stimulation. As shown in Fig. 2, the two straightchain hydroperoxides were more effective than $\mathrm{PGG}_{2}$ in triggering rapid oxygenation rates. It was not until $12 \mathrm{~s}$ after the addi- 
tion of $\mathrm{PGG}_{2}$ that the rate of oxygenation had accelerated to approximately the same magnitude achieved in $4 \mathrm{~s}$ with the other hydroperoxides.

Further insight into the specificity among stimulatory peroxides was obtained by comparing five different lipoxygenase-generated peroxides using the lagging system obtained with a cyanide-inhibited, crude enzyme preparation as described in Fig. 3. In this system, both the increased velocities at $9 \mathrm{~s}$ (Fig. 3A) and the decreased times to maximum velocity (Fig. $3 \mathrm{~B}$ ) were convenient indexes for designating peroxide stimulation (5). While each peroxide was quite active, the hydroperoxides of $18: 2(n-6)$ and 20:5(n-3) showed slightly greater potencies. However, overall there was no apparent correlation based on the position or number of double bonds.

To verify that the stimulation requires an intact hydroperoxide moiety, the effects of three different hydroperoxides and their corresponding hydroxy acids were compared. Using an assay system similar to that in Fig. 3, the hydroperoxides were effective activators, but the reduced compounds were ineffective (Fig. 4).

The stimulatory effects of hydrogen peroxide, t-butyl peroxide, and cumene hydroperoxide were determined to be much less as shown in Fig. 5. The concentrations required for half-maximal activation were

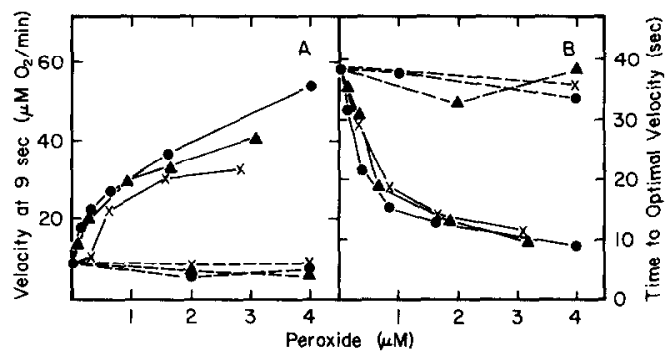

FIG. 4. Comparison of the activating effect of lipid hydroperoxides with that of their hydroxy acid derivatives. The velocity at $9 \mathrm{~s}$ (A) and time to optimum velocity (B) were measured, and lipid hydroperoxides were produced and assayed as described for Fig. 3 . Hydroxy acids were obtained by reduction of the lipid peroxide (generated with lipoxygenase) with $30 \mathrm{mg} \mathrm{NaBH}$ before extraction with ether. Hydroperoxy (solid lines) and hydroxy (dashed lines) acids were made from: $20: 3(n-6)(-\infty) ; 20: 3(n-3)$ $(\mathbf{\Delta}-\mathbf{\Delta}) ; 18: 3(n-3)(\times-\times)$.

approximately 50-fold higher than those for the peroxides tested in Fig. 4. Also, although cumene hydroperoxide reduced the time needed to attain maximum velocity, the velocity at $9 \mathrm{~s}$ could not be elevated to the extent achieved with the other peroxides.

While performing assays for peroxide activators, significant peroxide contamination of the stock solutions of the substrate 20:4 was frequently noticed. As mentioned above, DDC, a reagent known to remove

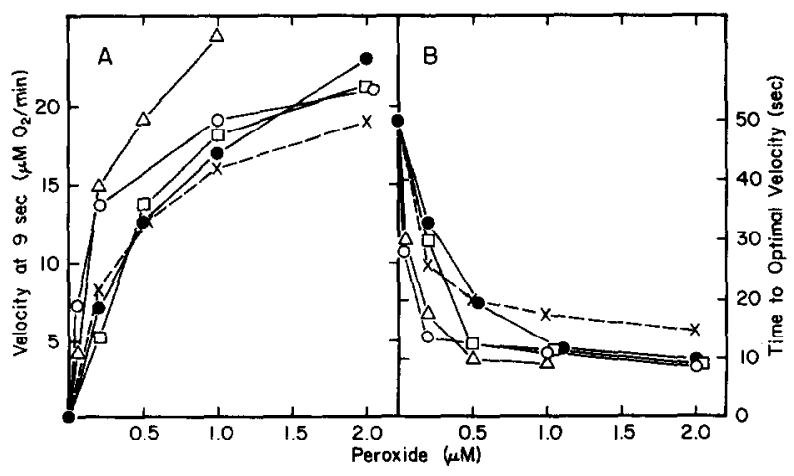

Fig. 3. Activation of prostaglandin cyclooxygenase by several fatty acid hydroperoxides. An aliquot of the lipid peroxide was added to $3.0 \mathrm{ml}$ of $0.1 \mathrm{M}$ Tris-chloride containing $0.67 \mathrm{mM}$ phenol, $50 \mu \mathrm{M}$ 20:4(n-6), and $1.5 \mathrm{mM} \mathrm{NaCN}$ in the oxygen electrode chamber. After $30 \mathrm{~s}, 0.38 \mathrm{mg}$ of phenol-activated vesicular gland acetone powder preparation was added to initiate the reaction. Lipid peroxides from the following fatty acids were tested: $20: 4(n-6)(\odot-\bigcirc) ; 18: 3(n-6)(\times---\times) ; 20: 2(n-6)(\square \square \square)$; $20: 5(n-3)(\mathrm{O}-\mathrm{O}) ; 18: 2(n-6)(\triangle \longrightarrow \triangle)$. 


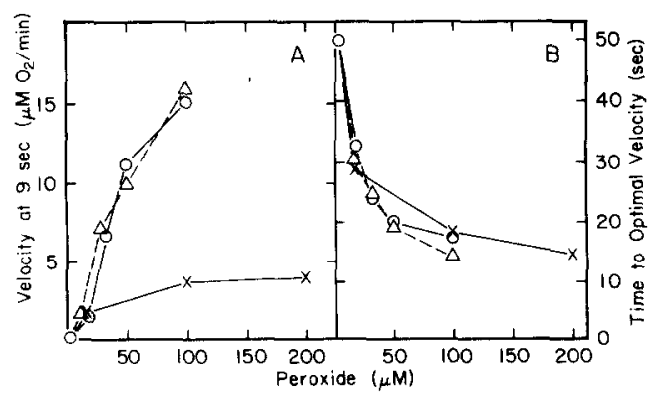

FiG. 5. Effectiveness of hydrogen peroxide, t-butyl hydroperoxide, and cumene hydroperoxide as activators of cyclooxygenase. Preparations of hydrogen peroxide $(\mathrm{O}-\mathrm{O})$, t-butyl hydroperoxide $(\Delta---\Delta)$, and cumene hydroperoxide $(x-x)$ were assayed for stimulatory activity as in Fig. 3 .

peroxide $(9,14)$, could remove these contaminants within about $3 \mathrm{~min}$. To directly demonstrate the spontaneous formation of stimulatory peroxides, solutions of 20:4 were allowed to autoxidize for various times and then assayed for peroxide content and stimulatory activity. An untreated $14 \mathrm{~mm}$ 20:4 solution, freshly prepared from the manufacturer (zero time), was contaminated with $0.69 \mathrm{mM}$ peroxide, and the peroxide level increased with time to a level of $1.6 \mathrm{~mm}$ at $72 \mathrm{~h}$. Figure 6 shows the simultaneous appearance of stimulatory activity and peroxide. Significantly, the peroxides from autoxidation were as stimulatory (at $0.25-$ $0.53 \mu \mathrm{M})$ as the 15-hydroperoxy-20:3 and $-20: 4$ tested in Fig. 2. In control experiments, $14 \mathrm{mM} \mathrm{20:4}$ that had been treated with sodium borohydride had no initially detectable peroxide and, with $0.2 \mathrm{~mm}$ butylated hydroxy toluene present, no peroxide was detected during $72 \mathrm{~h}$. The activation evidenced by the velocity at $6 \mathrm{~s}$ was no more than that of the blank solution of Tris buffer $\left(2.9 \mu \mathrm{M} \mathrm{O} \mathrm{O}_{2} / \mathrm{min}\right)$.

Results from thin-layer chromatography (solvent system was diethyl ether:methanol: acetic acid, 90:1:2) showed that oxygenated products (generated as described in the legend to Fig. 1) comigrated with $\mathrm{PGF}_{2 \alpha}$ (5\%, $R_{f}$ 0.06), $\mathrm{PGE}_{2}\left(54 \%, R_{f} 0.13\right), \mathrm{PGD}_{2}$ $\left(29 \% R_{f} 0.36\right)$, and $\mathrm{PGH}_{2}\left(11 \%, R_{f} 0.88\right)$. The stoichiometry of oxygen consumed per arachidonic acid was 2.06. Reaction conditions as described in the legend to Fig. 3

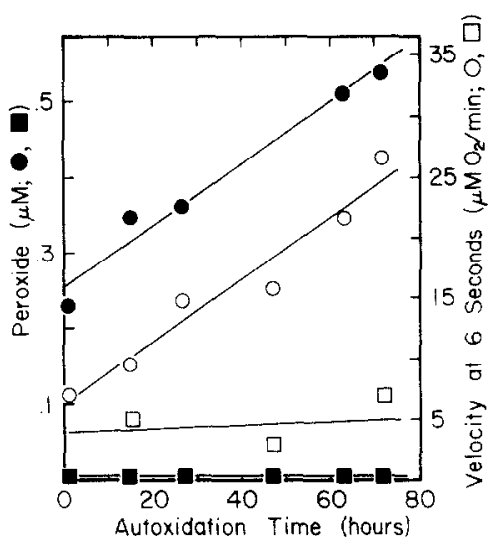

FIG. 6. Autoxidation of 20:4 generates stimulatory peroxides. Solutions of 20:4 in toluene were untreated, or treated with sodium borohydride and stored with $0.2 \mathrm{~mm}$ butylated hydroxytoluene (BHT). Aliquots were evaporated under nitrogen and made aqueous

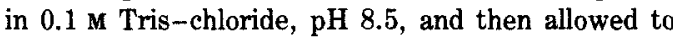
autoxidize at $25^{\circ} \mathrm{C}$ for varying times. Then untreated $(0,0)$ and borohydride/BHT-treated $(\square, \square)$ samples (14 mM) were analyzed for peroxide (closed symbols, see Materials and Methods) and $10-\mu 1$ aliquots of 10-fold diluted (3000-fold dilution overall) samples were analyzed for stimulatory activity as described for Fig. 1 (open symbols). The values listed for peroxide concentration ( $y$-axis) are 3000 -fold lower than in the stock $14 \mathrm{~mm}$ solutions and represent the final concentrations in the reaction mixtures used to assay for stimulatory activity.

yielded products which comigrated with $\mathrm{PGF}_{2 \alpha}\left(5 \%, R_{f} 0.27\right), \mathrm{PGE}_{2}\left(43 \%, R_{f} 0.39\right)$, $\mathrm{PGD}_{2}\left(35 \%, R_{f} 0.53\right)$, and $\mathrm{PGH}_{2}$-hydroxy acids $\left(17 \%, R_{f} 0.6-0.8\right)$ using a solvent system of chloroform:methanol:acetic acid: water (90:9:0.5:1:0.5). The observed product distributions are characteristic for the spontaneous nonenzymic rearrangement of the endoperoxide (15).

\section{DISCUSSION}

The wide variety of peroxides which activate the cyclooxygenase leads to several consequences linking the regulation of cellular peroxide levels to an indirect regulation of the production of prostaglandins by cyclooxygenase (Fig. 7). In the absence of activating levels of autoxidized fatty acid in vivo, peroxidatic or catalatic processes which remove peroxides may have an inhibitory effect by keeping the cyclooxygenase 


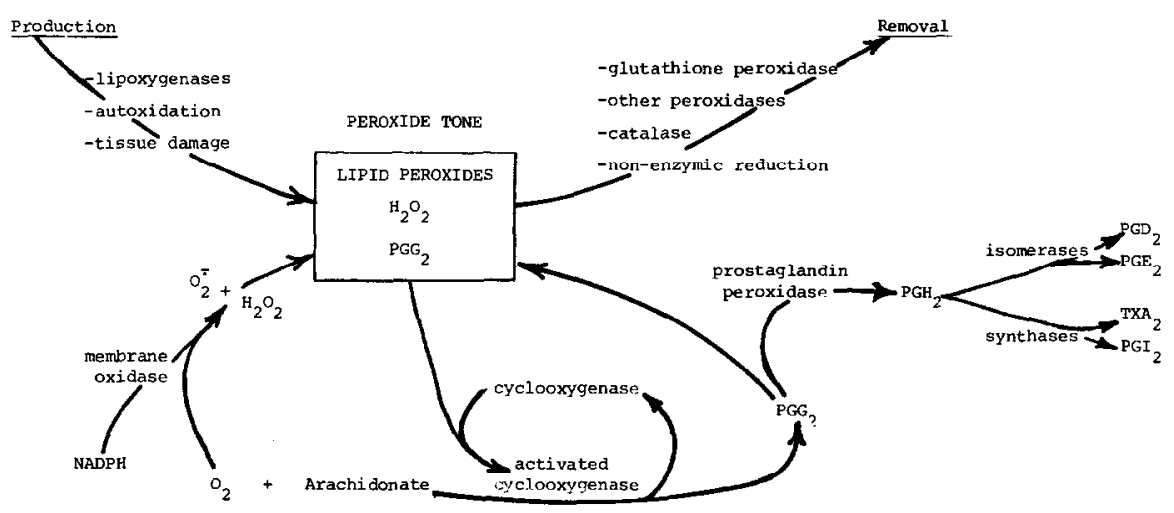

FIG. 7. Indirect regulation of prostaglandin biosynthesis by factors which regulate the peroxide tone.

in an inactive form. Inhibitory levels of glutathione peroxidase and its essential cofactor, glutathione, can be found in normal tissues [e.g., (16)]. In the presence of cosubstrates, any other peroxidase, including the cyclooxygenase peroxidase, would reduce the steady-state levels of peroxides. Also the weak triggering action of hydrogen peroxide would be prevented by catalase when present. This may be of some relevance in interpreting the reported inhibitory effect of catalase on platelet aggregation (17) and on microsomal cyclooxygenase (18). Besides enzymic removal of peroxides, agents which nonenzymically reduce peroxides (e.g., certain sulfhydryl compounds) or prevent the occurrence of peroxidation (antioxidants) may also contribute to a lowering of peroxide tone.

Since the product, $\mathrm{PGG}_{2}$, is an activator, it appears that once the reaction is triggered by small amounts of any hydroperoxide, an accelerative production of peroxide then results in continued activation. This concept provides a new hypothesis for the role of mammalian lipoxygenases (19-24) which generate lipid peroxides (22). Interestingly, an impaired platelet aggregation associated with a defective level of platelet lipoxygenase $(25,26)$ could be due to inadequate cyclooxygenase activity as a result of failure of the lipoxygenase to generate sufficient peroxide. Other processes, such as the stimulation of membrane NADH oxidase activity, could lead to the formation of stimulatory hydrogen peroxide. In fact, hydrogen peroxide has been reported to stimulate cyclooxygenase activity in mollusks (27). In addition, oxidase-generated $\mathrm{H}_{2} \mathrm{O}_{2}$ and $\mathrm{O}_{\overline{\mathbf{z}}}$ can initiate membrane lipid oxidation leading to the formation of stimulatory levels of peroxidized lipids. The above hypothesis regarding peroxide tone suggests that further studies of phagocytic cells at inflammatory sites or during immune responses may show that their production of oxidants and lipid peroxides facilitates local prostaglandin biosynthesis.

\section{ACKNOWLEDGMENTS}

This work was supported in part by National Science Foundation Grant BMS-75-13157, a Canadian Medical Research Council fellowship (H.W.C.), and an Upjohn Graduate Research fellowship (M.E.H.).

\section{REFERENCES}

1. Nugteren, D. H. (1975) J. Biol. Chem. 250, 2808-2812.

2. Wolfe, L. S., Rostworowski, K., AND PaPPIUS, H. M. (1976) Canad. J. Biochem. 54, 629-640.

3. Lands, W. E. M., Lee, R., and Smith, W. (1971) Ann. N. Y. Acad. Sci. 180, 107-122.

4. SMith, W. L., AND LaNDS, W. E. M. (1972) Biochemistry 11, 3276-3285.

5. CoOK, H. W., AND LANDS, W. E. M. (1975) Biochem. Biophys. Res. Commun. 65, 464-471.

6. HemLer, M. E., LaNDS, W. E. M., AND GRAFF, G. (1978) Biochem. Biophys. Res. Commun. 85, $1325-1331$.

7. LaNDS, W. E. M., CoOK, H. W., AND RoMe, L. H. (1976) in Advances in Prostaglandin and Thromboxane Research (Samuelsson, B., and Paoletti, R., eds.), Vol. I, pp. 7-17, Raven Press, New York. 
8. Hemler, M., Lands, W. E. M., and Smith, W. L. (1976) J. Biol. Chem. 251, 5575-5579.

9. Hemler, M. E., Crawford, C. G., and LaNds, W. E. M. (1978) Biochemistry 17, 1772-1779.

10. Wallach, D. P., AND Daniels, E. G. (1971) Biochim. Biophys. Acta 231, 445-457.

11. Rome, L. H., AND Lands, W. E. M. (1975) Prostaglandins 10, 813-824.

12. Cook, H. W., Ford, G., AND Lands, W. E. M. (1979) Anal. Biochem., in press.

13. Cook, H. W., AND LaNDS, W. E. M. (1975) Canad. J. Biochem. 53, 1220-1231.

14. Ishimura, Y., AND Hayaishi, O. (1973) J. Biol. Chem. 248, 8610-8612.

15. Hamberg, M., and Samuelsson, B. (1973) Proc. Nat. Acad. Sci. USA 70, 899-903.

16. Cook, H. W., AND LANDS, W. E. M. (1976) Nature (London) 260, 630-632.

17. Vargaftig, B. B., Tranier, Y., and Chignard, M. (1975) Eur. J. Pharmacol. 33, 19-29.

18. Panganamala, R. V., Sharma, H. M., SPrecher, H., GeEr, J. C., AND CoRnwell, D. G. (1974) Prostaglandins 8, 3-11.
19. Hamberg, M. (1976) Biochim. Biophys. Acta 431, 651-654.

20. Borgeat, P., Hamberg, M., and Samuelsson, B. (1976) J. Biol. Chem. 251, 7816-7820.

21. Hamberg, M., and Samuelsson, B. (1974) Proc. Nat. Acad. Sci. USA 71, 3400-3404.

22. Nugteren, D. H. (1975) Biochim. Biophys. Acta 380, 299-307.

23. Ho, P. P. K., Walters, P., and Sullivan, H. R. (1977) Biochem. Biophys. Res. Commun. 76, 398-404.

24. Hammarstrom, S., Hamberg, M., Samuelsson, B., Duell, E. A., Stawiski, M., AND Voorhees, J. J. (1975) Proc. Nat. Acad. Sci. USA $72,5130-5134$.

25. OKUma, M., and UChina, H. (1977) N. Engl. $J$. Med. 297, 1351-1352.

26. Keenan, J. P., Wharton, J., Shepherd, A. J. N., AND Bellingham, A. J. (1977) Brit. J. Haematol. 35, 275.

27. Morse, D. E., Duncan, H., Hooker, N., AND Morse, A. (1977) Science 196, 298-300.

28. Hemler, M. E., AND Lands, W. E. M. (1977) Lipids 12, 591-595. 